\title{
Características de crecimiento y medidas del área del ojo del lomo en ovinos criollos del departamento de Córdoba, Colombia
}

\section{Growth traits and measures of loin eye area in Creole sheep of the Cordoba department, Colombia}

\author{
Oscar Vergara Garay ${ }^{1}$; Juan Simanca Sotelo ${ }^{2}$; Moris Bustamante Yánez ${ }^{3}$; José Bula Torres ${ }^{4}$; \\ Camilo Camargo Pitalua ; ${ }^{5}$ Nassif Mahuad Ruíz ${ }^{6}$
}

'Zoot, Dr Sc. Universidad de Córdoba, Facultad de Medicina Veterinaria y Zootecnia, Grupo de Investigación en Producción Animal Tropical. Montería Córdoba, Colombia; e-mail: overgara@correo.unicordoba.edu.co; Dhttps://orcid.org/0000-0002-1027-9469

${ }^{2}$ MVZ. M.Sc. Universidad de Córdoba, Facultad de Medicina Veterinaria y Zootecnia, Grupo de Investigación en Producción Animal Tropical. Montería Córdoba, Colombia; e-mail: jcsimanca@correo.unicordoba.edu.co; Dhttps://orcid.org/0000-0002-3744-7055

${ }^{3}$ MVZ, M.Sc. Universidad de Córdoba, Facultad de Medicina Veterinaria y Zootecnia, Grupo de Investigación en Producción Animal Tropical. Montería Córdoba, Colombia; e-mail: morisbustamante@correo.unicordoba.edu.co; Dhttps://orcid.org/0000-0003-2874-2025

${ }^{4}$ Est. MVZ. Universidad de Córdoba, Facultad de Medicina Veterinaria y Zootecnia, Programa de Medicina Veterinaria y Zootecnia. Montería - Córdoba, Colombia; e-mail: djb9612@outlook.com; Dhttps://orcid.org/0000-0003-3623-4370

${ }^{5}$ Est. MVZ. Universidad de Córdoba, Facultad de Medicina Veterinaria y Zootecnia, Programa de Medicina Veterinaria y Zootecnia. Montería - Córdoba, Colombia; e-mail: ccamargopitalua60@correo.unicordoba.edu.co; (Dhttps://orcid.org/0000-0003-0910-7800

${ }^{6}$ Est. MVZ. Universidad de Córdoba, Facultad de Medicina Veterinaria y Zootecnia, Programa de Medicina Veterinaria y Zootecnia. Montería - Córdoba, Colombia; e-mail: nmahuadruiz@correo.unicordoba.edu.co; Dhttps://orcid.org/0000-0001-7298-9135org/0000-0003-2911-0871

Cómo citar: Vergara Garay, O.; Simanca Sotelo, J.; Bustamante Yánez, M.; Bula Torres, J.; Camargo Pitalua, C.; Mahuad Ruíz, N. 2019. Características de crecimiento y medidas del área del ojo del lomo en ovinos criollos del departamento de Córdoba, Colombia. Rev. U.D.C.A Act. \& Div. Cient. 22(2):e1371. http://doi.org/10.31910/rudca.v22.n2.2019.1371

Artículo de acceso abierto publicado por Revista U.D.C.A Actualidad \& Divulgación Científica, bajo una licencia Creative Commons CC BY-NC 4.0

Recibido: Agosto 21 de 2018

Aceptado: Octubre 31 de 2019

Editado por: Ingeborg Zenner de Polanía

\section{RESUMEN}

De la raza ovina criolla, desarrollada en el departamento de Córdoba, Colombia, existen pocas investigaciones sobre características de desarrollo, por tal razón, el objetivo del presente estudio fue evaluar características asociadas al crecimiento de los ovinos criollos de pelo, en dos poblaciones de Córdoba, Colombia. Se utilizaron 55 animales provenientes de dos sistemas de producción. El pesaje, se realizó cada 15 días, desde el nacimiento hasta los 6 meses de edad y las mediciones del área del ojo del lomo (AOL), se tomaron por ultrasonografía, a los 4 y 6 meses de edad. Las características evaluadas fueron peso al nacer (PN), peso al destete (PDA90), peso a los seis meses (PA6M), ganancia de peso predestete (GPPRD) y posdestete (GPPOD) y AOL. El promedio para PN fue de 2,63 \pm 0,65 kg; para PDA90, de 11,19 $\pm 3,78 \mathrm{~kg}$; para PA6M, de 15,61 \pm $3,93 \mathrm{~kg}$ y para GPPRD y GPPOD, de $0,095 \pm 0,04$ y $0,06 \pm 0,3$ 
$\mathrm{kg} / \mathrm{d}$, respectivamente. Se encontró que el sexo del cordero afectó $(\mathrm{P} \leq 0,05)$ las variables PDA90, PA6M y GPPRD. El tipo de finca tuvo influencia $(\mathrm{P} \leq 0,05)$ sobre todas las características de crecimiento evaluadas. Los resultados sugieren que habría que mejorar los sistemas de alimentación en las poblaciones estudiadas e iniciar un proceso de selección, con el fin de mejorar las características evaluadas.

Palabras clave: peso al nacer; peso al destete; ganancia de peso; ovinos criollos; canal.

\section{ABSTRACT}

The Creole sheep breed developed in the department of Córdoba, Colombia has little research on growth traits, for this reason the objective of this study was to evaluate traits associated with the growth of Creole hair sheep in two populations of Córdoba, Colombia. Fifty-five Creole animals from two production systems were used. Weighing was performed every 15 days from birth to 6 months of age and loin eye area (LEA) measurements were taken by ultrasound at 4 and 6 months of age. The traits evaluated were birth weight (BW), weaning weight (WW90), weight at six months of age (W6M), preweaning (PWWG) and postweaning weight gain (POWWG) and LEA. The average for BW was $2.63 \pm 0.65 \mathrm{~kg}$, for WW90 it was $11.19 \pm 3.78 \mathrm{~kg}$, for W6M it was $15.61 \pm 3.93 \mathrm{~kg}$ and for PWWG and POWWG it was $0.095 \pm 0.04$ and $0.06 \pm 0.03 \mathrm{~kg} / \mathrm{d}$, respectively. It was found that the sex of the lamb affected $(\mathrm{P} \leq 0.05)$ the variables PDA90, PA6M and GPPRD. The type of farm had influence $(\mathrm{P} \leq 0.05)$ on all the growth traits evaluated. The results suggest that, it would be necessary to improve the feeding systems in the populations studied and initiate a selection process in order to improve the evaluated traits.

Keywords: birth weight; weaning weight; weight gain; sheep creole; carcass.

\section{INTRODUCCIÓN}

Los diferentes sistemas de producción pecuarios tienen como objetivo común la producción de alimentos para el hombre. Dentro de estos sistemas, se puede encontrar la explotación ovina que, en los últimos años en Colombia, ha despertado el interés por parte de los productores, por ser animales de tamaño mucho menor que el bovino y de fácil manejo, además de producir carne y leche para la humanidad. En el departamento de Córdoba, la ovino-cultura se encuentra en etapa de crecimiento, pasando su población de 22.783 ovinos en el 2014, a 104.604, en el 2019 (DANE, 2016; ICA, 2019), en donde los ovinos se han convertido en una fuente de trabajo y de producción y está dejando de ser una especie relegada en comparación con los sistemas de producción bovina.

Para sacar un mayor provecho al ovino criollo nativo, se tienen que llevar a cabo investigaciones que muestren los pros y los contras que brindarían estos animales al sistema productivo, debido a que, la información sobres ellos, es escasa. Además, al ser un sistema que anteriormente se manejaba de manera extensiva y sin ningún soporte técnico, el mejoramiento que se ha hecho en las características de importancia para la producción es poco. El estudio de las características de crecimiento es necesario para implementar con un adecuado programa de mejoramiento genético, que tiene como meta aumentar, la precocidad de la especie, además que sirve para inferir las tasas de crecimiento, necesidades alimenticias, pesos y grado de madurez de cada especie (Lobo et al. 2006). Las medidas de crecimiento las han utilizado algunos investigadores como guía para describir el crecimiento y el estudio de características de interés económico, como indicadores de peso vivo y para evaluar el desarrollo muscular y grado de acabado de los animales (Lobo et al. 2006; Fitzhugh, 1976).

Cabe resaltar que existen factores que influyen sobre el crecimiento de los ovinos, como la edad, la raza, el tipo de animal, la fase fisiológica, el estado nutricional, el tipo de nacimiento y las estrategias de manejo (Patiño \& Van Cleef, 2010).

Por otro lado, la medición del área del ojo del lomo (AOL), mediante ultrasonografía, es una opción práctica a bajo costo, que permite evaluar el mérito de la carcasa en el animal vivo (Battista et al. 2017), donde el aumento del área del ojo del lomo es considerado el objetivo más importante de la industria cárnica ovina para optimar el mérito de la canal, mejorando esta, si los animales se sacrifican a una edad más temprana (Lupton, 2008).

Por ello, el objetivo de este estudio fue evaluar características asociadas al crecimiento, así como el área del ojo del lomo de los ovinos criollos de pelo, en Córdoba, Colombia, como herramienta para volver eficientes y competitivos a los sistemas de producción ovina en la región.

\section{MATERIALES Y MÉTODOS}

Localización. Los datos utilizados en este estudio fueron obtenidos de dos sistemas de producción ovina, en los municipios de Montería y Sahagún, departamento de Córdoba. El municipio de Montería está localizado al noroeste de Colombia, a $8^{\circ} 45^{\prime}$ de latitud Norte y $75^{\circ} 53^{\prime}$ de longitud Oeste. Tiene una altitud de $18 \mathrm{~m}$ s.n.m., una temperatura promedio de $28^{\circ} \mathrm{C}$, una humedad relativa del $85 \%$ y una precipitación media anual de $1.156 \mathrm{~mm}$. Por su parte, el municipio de Sahagún, se encuentra ubicado en la parte nororiental del departamento de Córdoba, se localiza a los 856 $58^{\prime \prime}$ ' de latitud Norte y $75^{\circ} 26^{\prime} 52^{\prime \prime}$ de longitud Oeste, a $71 \mathrm{~km}$ de Montería, a $75 \mathrm{~m}$ s.n.m. y posee una temperatura media de $26,9^{\circ} \mathrm{C}$ (Santana, 1999).

Las madres y los corderos al destete fueron manejados bajo condiciones de pastoreo en praderas de Bothriochloa pertusa, con disponibilidad de agua y sal mineral a libre acceso; durante la época seca, los animales del sistema de producción de Montería se suplementaron con ensilado de maíz (Zea mays) con grano y los del sistema de producción de Sahagún, con ensilado de Pennisetum purpureum.

Los animales, se manejaron por monta estacional, en el cual, las hembras que se expusieron a macho por un periodo de 45 días, 
posibilitando la oportunidad de cubrir dos ciclos ováricos de la hembra, durante dicha estación.

Desde el punto de vista sanitario, las hembras fueron sometidas a una evaluación sanitaria, específicamente, evaluando puntaje de FAMACHA y la carga parasitaria, mediante técnica de Mac Master. Luego, se realizó desparasitación selectiva y control de la carga parasitaria a los 15 días de haber realizado desparasitación. Un mes antes del parto, los lotes de hembras reproductoras fueron desparasitadas y vacunadas contra clostridiosis y pasteurelosis. Las crías evaluadas fueron vacunadas 15 días antes del destete contra clostridiosis y pasteurelosis, y revacunadas a los 15 días.

Recolección y organización de datos. Se utilizaron 55 corderos, provenientes de 55 hembras y cuatro machos reproductores, dos en cada finca. En el sistema de producción de Montería, se utilizaron 15 (9 machos y 6 hembras) y en el sistema de producción de Sahagún, 40 animales (18 machos y 22 hembras); los animales nacieron entre noviembre del 2013 y abril del 2014. Al nacimiento de los animales fueron identificados con collares y permanecieron con sus madres hasta los 90 días, momento en que se realizó el destete. Se realizaron pesajes cada 15 días, a partir del nacimiento hasta los 6 meses de edad y mediciones del área del ojo del lomo (AOL) por ultrasonografía, a los 4 y 6 meses de edad. Las mediciones de peso en cada una de los sistemas de producción, se obtuvieron con una báscula digital JAZDINA L150, con capacidad para 150kg; además, se realizó el registro de cada pesaje, donde se tuvo en cuenta: la identificación del animal, la fecha y edad del animal, la fecha del parto, la mortalidad en el periodo pre y post-destete, el sexo de los animales y la identificación de los padres. Todos los animales provenían de partos simples y no se disponía de información precisa del número de partos de la madre. El peso de los animales fue ajustado al destete (90 días) y a los seis meses utilizando la siguiente fórmula:

$$
P_{x i}=\frac{\left(P_{y i}-P N\right)}{y i}+P N
$$

Donde:

$\mathrm{P}_{\mathrm{xi}}=$ Peso estimado para la edad $x i$, siendo $x i=90$ y 180 días de edad.

$\mathrm{P}_{\mathrm{yi}}=$ Peso tomado a la edad más próxima de xi; $y i=$ edad en días, más próxima de $x i$.

$\mathrm{PN}=$ Peso al nacimiento.

Para determinar el AOL, se utilizó un ecógrafo marca ALOKA SSD500 , equipado con transductor lineal de $5 \mathrm{Mhz}$. Antes de la captura de imágenes por el ecógrafo, se procedió a limpiar la región entre la $12^{\mathrm{a}}$ y $13^{\mathrm{a}}$ vértebras torácicas del lado izquierdo del animal, seguido del afeitado de esta zona, para evitar interferencia de la transmisión del haz de la onda de sonido.

La sonda estaba equipada con una guía acústica, que se colocó de manera perpendicular al músculo Longissimus dorsi, entre la $12^{\mathrm{a}}$ y $13^{\mathrm{a}}$ vértebra torácica, para medir el área del AOL. Las imágenes obtenidas fueron analizadas con el analizador de imágenes Eview-
Echo ImageViewer y la información proveniente de estas medidas, se anotó en registros diseñados para tal fin.

Análisis estadístico. Las características evaluadas fueron peso al nacer (PN), peso al destete (PDA90), peso a los seis meses de edad (PA6M), ganancia de peso predestete (GPPRD) y posdestete (GPPOD) y área del ojo del lomo (AOL), a las cuales, se les realizó estadística descriptiva. Además, se efectuó un análisis de varianza por medio del procedimiento GLM del SAS (SAS, 2007) y para determinar diferencias significativas entre sexo y finca para las variables en estudio, se utilizó el procedimiento LSMEANS (SAS, 2007).

\section{RESULTADOS Y DISCUSIÓN}

En la tabla 1, se muestran la estadística descriptiva de las diferentes características de crecimiento evaluadas. La media del PN fue similar a la reportada por López-Ordaza et al. (2012), en ovinos criollos de Chiapas $(2,48 \pm 0,52)$ y por Ríos-Utrera et al. (2014), en raza Pelibuey $(2,6 \pm 0,07)$. Un promedio superior fue encontrado por Raoof et al. (2017), en ovinos nativos en Kurdistan (3,9); Mortimer et al. (2017), en animales Merino en Australia (4,58 $\pm 1,08)$; Baneh \& Ahmadpanah (2018), en ovinos Ghezel en Irán (4,27 \pm 0,68); Haile et al. (2018) y Jawasreh et al. (2018), en animales Awassi en Jordán $(4,48 \pm 0,8$ y 4,54 $\pm 0,75)$. Por su parte, un PN inferior al de este estudio fue reportado por Macedo \& Arredondo (2008), en ovinos de Pelibuey $(2,30 \pm 0,11)$ y Safi et al. (2017), en ovinos Harnai $(2,39$ $\pm 0,23)$. Cabe resaltar que las diferencias entre los pesos al nacer del presente estudio con la literatura citada, se debe a las diferencias raciales, de alimentación y manejo de cada una de las poblaciones, que van a influir sobre esta característica.

Con relación al PDA90, el promedio estimado se encuentra por encima del reportado por López-Ordaza et al. (2012), en México $(8,96 \pm 2,54)$ y es similar al obtenido por Hinojosa-Cuéllar et al. (2012), en corderos Pelibuey, en México $(11,0 \pm 0,09)$. Un promedio superior fue encontrado por Safi et al. (2017), Mortimer et al. (2017) y Baneh \& Ahmadpanah (2018), a los 90 días de destete (13,51 0 0,38, $23,8 \pm 5,06$ y 23,37 $\pm 4,12$ ), Jawasreh et al. (2018) y Haile et al. (2018), a una edad de destete de $65(17,13 \pm 0,7)$ y $56(19,34 \pm 4,50)$ días. $\mathrm{Al}$ igual que el PN, las diferencias de PDA90 entre las poblaciones citadas y este estudio, se deben a diferencias raciales, de alimentación y de manejo.

Para PA6M, la media estimada se encuentra por debajo de la reportada por Quintero et al. (1997) y Dickson-Urdaneta et al. (2004), en la raza West African (17,0 \pm 1,34 y 18,0 \pm 4,5); por Maza et al. (2015), en animales criollos $(23,6 \pm 3,7)$ y por Baneh \& Ahmadpanah (2018), en Merino (31,83 $\pm 5,32)$. Para mejorar el peso a los seis meses, que es el peso que, por lo general, los productores venden sus animales al mercado para el sacrifico, se debe mejorar la alimentación de los animales, especialmente, en el periodo posdestete y comenzar a realizar procesos de selección para las características de crecimiento en los ovinos criollos de pelo. 
Tabla 1. Resultados de estadística descriptiva para las características de crecimiento evaluadas.

\begin{tabular}{|l|c|c|c|c|c|}
\hline Variable & $\mathbf{n}$ & Media & Desviación estándar & Min & Max \\
\hline PN (kg) & 55 & 2,63 & 0,65 & 1,50 & 4,00 \\
\hline PDA90 (kg) & 55 & 11,19 & 3,78 & 4,84 & 20,00 \\
\hline PA6M (kg) & 37 & 15,61 & 3,93 & 10,50 & 27,66 \\
\hline GPPRD (g/d) & 55 & 0,095 & 0,04 & 0,03 & 0,19 \\
\hline GPPOD (g/d) & 37 & 0,060 & 0,03 & $-0,005$ & 0,11 \\
\hline
\end{tabular}

PN: Peso al nacimiento; PDA90: Peso al destete ajustado a 90 días; PA6; Peso ajustado a los seis meses; GPPRD: Ganancia de peso predestete; GPPOD: Ganancia de peso posdestete.

Para GPPRD, el promedio estimado $(0,095 \pm 0,04 \mathrm{~kg} / \mathrm{d})$ fue inferior al encontrado por González et al. (2002), en ovinos Black Belly $(0,122 \pm 0,026)$; Dickson-Urdaneta et al. (2004), en animales West African (0,101 $\pm 0,03)$; Jawasreh et al. (2018) y Haile et al. (2018), en ovinos Awassi $(0,20 \pm 0,07$ y $0,264 \pm 0,046)$ y por Ríos-Utrera et al. (2014), en cruces Dorper x Black Belly $(0,102 \pm 0,005)$ y Katahdin x Pelibuey $(0,104 \pm 0,004)$. Un promedio similar al de este estudio fue hallado por Ríos-Utrera et al. (2014), en ovinos Dorper x Pelibuey $(0,097 \pm 0,005)$, Katahdin x Black Belly $(0,095 \pm 0,005)$ y Pelibuey $(0,093 \pm 0,004)$. Por su parte, Ríos-Utrera et al. (2014) encontraron un valor inferior en ovinos Pelibuey x Black Belly $(0,085$ $\pm 0,005)$. Con relación a la GPPOD, el promedio encontrado $(0,06$ $\pm 0,03 \mathrm{~kg} / \mathrm{d}$ ) fue similar al hallado por Dickson-Urdaneta et al. (2004) $(0,069 \pm 0,03)$ y Maza et al. (2015) $(0,064,68 \pm 0,008)$, en ovinos criollos suplementados e inferior al reportado por González et al. (2002) $(0,088 \pm 0,054)$.

El sexo del cordero tuvo influencia $(\mathrm{P} \leq 0,05)$ sobre PDA90, PA6M y GPPRD, obteniendo mejor desempeño los machos, con relación a las hembras.

Según la investigación realizada por Hinojosa-Cuéllar et al. (2012), ninguna de las tres variables dependientes (PN, GPPRD y PD) estudiadas fue afectada por el sexo $(p>0,05)$ de la cría en ovinos de raza Pelibuey, similar a lo reportado por Benyi et al. (2006), en corderos híbridos West African; sin embargo, Carrillo \& Segura (1993), en corderos Pelibuey, encontraron que los machos tenían valores más altos en PN y PD que las hembras; asimismo, Jawasreh et al. (2018), en ovinos Awassi, reportaron efecto del sexo sobre el peso al nacer, al destete y en la GPPRD. De acuerdo con Bores $e t$ al. (2002), el efecto hormonal de la testosterona en los corderos machos no se manifiesta significativamente a edades inferiores a 70d. Posterior a ello, la testosterona promueve un crecimiento mayor en los machos con relación a las hembras, lo que explica las diferencias en los pesos para PDA90 y PA6M.
El tipo de finca afectó $(P \leq 0,05)$ todas las características de crecimiento evaluadas (Tabla 2). Las diferencias encontradas entre las medias de los pesos de las características de crecimiento evaluadas, se podría deber al manejo que se brinda en cada finca a los individuos dedicados a la producción, básicamente al alimento que se les proporciona a estos. De acuerdo con los resultados encontrados en este estudio, como se mencionó anteriormente, habría que mejorar los sistemas de alimentación en las poblaciones estudiadas e iniciar un proceso de selección, con el fin de mejorar los promedios de las características evaluadas.

Con relación al AOL, el sexo del cordero no afectó $(\mathrm{p}>0,05)$ el AOL a los cuatro y seis meses de edad de los corderos. Por su parte, el factor finca influyó $(\mathrm{p} \leq 0,05)$ a los cuatro meses de edad (Tabla 3). Varios autores han encontrado medidas superiores para el AOL; es así como Bianchi (2006) reportó valores de 14,39, 14,24 y 13,89 $\mathrm{cm}^{2}$, en ovinos de 140 días de edad, de las razas Merino, Mestizo y Suffolk. Por su parte, Kiyanzad (2004) consiguió un promedio de AOL al año de edad de 11,39 y $10,90 \mathrm{~cm}^{2}$, en ovinos Moghani y Makui; Correa Santos et al. (2013), en ovinos Santa Inés castrados, que recibieron diferentes tipos de suplementación, encontraron promedios entre 11,16 y $12,77 \mathrm{~cm}^{2}$; Battista et al. (2017) registraron, en corderos de 200 días de edad, un promedio de $11,16 \pm 0,21 \mathrm{~cm}^{2}$. La diferencia que existe entre los resultados de este estudio para el AOL y la literatura citada, está relacionada con la poca selección que se ha sometido el ovino criollo, con relación a otras razas, que han sido mejoradas genéticamente para la producción de carne. Hay que tener claro que un ojo de lomo más grande tiende a aumentar el rendimiento valor de la canal (Leeds et al. 2008; Notter et al. 2014).

Conflicto de intereses: El manuscrito fue preparado y revisado con la participación de todos los autores, quienes declaramos que no existe conflicto de intereses que ponga en riesgo la validez de los resultados presentados. Financiación. Esta investigación fue financiada por la Vicerrectoría de Investigación y Extensión de la Universidad de Córdoba 
Tabla 2. Promedios para PN, PDA y P6, GPPRE, GPPOS, según el sexo y finca en ovinos Criollo.

\begin{tabular}{|c|c|c|c|c|}
\hline Variable & & & Media & P. Valor \\
\hline \multirow[t]{4}{*}{ PN } & Sexo & Macho & $2,89 \pm 0,12$ & 0,0621 \\
\hline & & Hembra & $2,57 \pm 0,13$ & \\
\hline & Finca & Montería & $2,97 \pm 0,15$ & 0,0146 \\
\hline & & Sahagún & $2,50 \pm 0,09$ & \\
\hline \multirow[t]{4}{*}{ PDA90 } & Sexo & Macho & $13,38 \pm 0,55$ & 0,0088 \\
\hline & & Hembra & $11,33 \pm 0,57$ & \\
\hline & Finca & Montería & $14,90 \pm 0,72$ & $<, 0001$ \\
\hline & & Sahagún & $9,82 \pm 0,44$ & \\
\hline \multirow[t]{4}{*}{ PA6M } & Sexo & Macho & $18,85 \pm 0,7$ & 0,0383 \\
\hline & & Hembra & $16,88 \pm 0,8$ & \\
\hline & Finca & Montería & $21,25 \pm 1,12$ & $<, 0001$ \\
\hline & & Sahagún & $14,49 \pm 0,48$ & \\
\hline \multirow[t]{4}{*}{ GPPRD } & Sexo & Macho & $0,11 \pm 0,005$ & 0,0175 \\
\hline & & Hembra & $0,09 \pm 0,006$ & \\
\hline & Finca & Montería & $0,13 \pm 0,01$ & $<, 0001$ \\
\hline & & Sahagún & $0,08 \pm 0,004$ & \\
\hline \multirow[t]{4}{*}{ GPPOD } & Sexo & Macho & $0,07 \pm 0,01$ & 0,5112 \\
\hline & & Hembra & $0,07 \pm 0,01$ & \\
\hline & Finca & Montería & $0,09 \pm 0,01$ & 0,0047 \\
\hline & & Sahagún & $0,05 \pm 0,004$ & \\
\hline
\end{tabular}

PN: Peso al nacimiento; PDA90: Peso al destete ajustado a 90 días; PA6; Peso ajustado a los seis meses; GPPRD: Ganancia de peso predestete; GPPOD: Ganancia de peso posdestete.

Tabla 3. Medias de AOL, según efectos sexo y finca.

\begin{tabular}{|c|c|c|c|c|c|}
\hline \multirow{2}{*}{\multicolumn{2}{|c|}{$\begin{array}{c}\text { Efecto } \\
\text { Media }\left(\mathrm{cm}^{2}\right)\end{array}$}} & \multicolumn{2}{|c|}{4 meses } & \multicolumn{2}{|c|}{6 meses } \\
\hline & & \multirow{2}{*}{$\frac{\text { D.E }\left(\mathbf{c m}^{2}\right)}{8,13^{a}}$} & \multirow{2}{*}{$\frac{\operatorname{Media}\left(\mathrm{cm}^{2}\right)}{0,54}$} & \multirow{2}{*}{$\begin{array}{c}\text { D.E }\left(\mathbf{c m}^{2}\right) \\
9,19^{a}\end{array}$} & \multirow[b]{2}{*}{0,65} \\
\hline \multirow{2}{*}{ Sexo } & Macho & & & & \\
\hline & Hembra & $8,03^{a}$ & 0,54 & $8,39^{\mathrm{a}}$ & 0,67 \\
\hline \multirow{2}{*}{ Finca } & 1 & $9,15^{a}$ & 0,54 & $9,38^{a}$ & 0,80 \\
\hline & 2 & $7,17^{\mathrm{b}}$ & 0,54 & $8,07^{\mathrm{a}}$ & 0,54 \\
\hline
\end{tabular}

Para un efecto determinado, letras diferentes en la misma columna, indica diferencia significativa (P $\leq 0,05)$. AOL: Área del ojo del lomo.

\section{REFERENCIAS}

1. BANEH, H.; AHMADPANAH, J. 2018. Genetic evaluation of body weight traits in Iranian native Ghezel sheep. Genetika. 50(1):275-284. https://doi.org/10.2298/GENSR1703791F

2. BATTISTA, S.; HOLÁSKOVÁ, I.; PRITCHARD, J.; INSKEEP, K. 2017. Effects of weight, age and breed type on loin eye area, loin depth and backfat thickness in replacement ewe lambs. Sheep \& Goat Res. J. 32(1):13-20.

3. BENYI, K.; NORRIS, D.; KARBO, N.; KGOMO, K. 2006. Effects of genetic and environmental factors on pre- weaning and post-weaning growth in West African crossbred sheep. Trop. Anim. Health Prod. 38(7):547-554. https://doi.org/10.1007/s11250-006-4416-2

4. BIANCHI, G. 2006. Alternativas tecnológicas para la producción de carne ovina de calidad en sistemas pastoriles. Editorial Hemisferio Sur. Uruguay. 283p.

5. BORES, F.; VELÁZQUEZ, A.; HEREDIA, A. 2002. Evaluación de razas terminales en esquemas de cruza comercial con ovejas de pelo F1. Tec. Pecu. Méx. 40(1):71-79. 
6. CARRILLO, A.; SEGURA, J. 1993. Environmental and genetic effects on preweaning growth performance of hair sheep in México. Trop. Anim. Health Prod. 25(3):173-178.

7. CORREA SANTOS, V.; BERTOCCO EZEQUIEL, J.M.; MORGADO SEVERNO, E. DA SILVA; DE SOUSA Jr., C. 2013. Carcass and meat traits of lambs fed by-products from the processing of oil seeds. Acta Scientiarum Animal Scie. 35(4):387-394. https://doi.org/10.4025/ actascianimsci.v35i4.20403

8. DEPARTAMENTO ADMINISTRATIVO NACIONAL DE ESTADÍSTICA, DANE. 2016. $3^{\circ}$ Censo Nacional Agropecuario. GIT Área de Comunicación DANE. Bogotá (Colombia). p.378. Disponible desde Internet en: https:// www.dane.gov.co/files/images/foros/foro-de-entregade-resultados-y-cierre-3-censo-nacional-agropecuario/ CNATomo2-Resultados.pdf (con acceso el 28/10/2019).

9. DICKSON-URDANETA, L.; TORRES-HERNÁNDEZ, G.; DÁUBETERRE, R.; GARCÍA, O. 2004. Crecimiento en ovinos West African bajo un sistema de pastoreo restringido en Venezuela. Rev. Fac Agron. 21(1):59-67.

10. FITZHUGH, H.A. 1976. Analysis of growth curves and strategies for altering their shape. J. Anim. Sci. 42(4):10361051.

11. GONZÁleZ, R.; TORRES, G.; CASTILLO, M. 2002. Crecimiento de corderos Blackbelly entre el nacimiento y el peso final en el trópico húmedo de México. Vet. Méx. 33(4):443-453.

12. HAILE, A.; HILALI, M.; HASSEN, H.; LOBO, H.R.N.B.; RISCHKOWSKY, B. 2018. Estimates of genetic parameters and genetic trends for growth, reproduction, milk production and milk composition traits of Awassi sheep. Animal. 13(2):1-8. https://doi.org/10.1017/ S1751731118001374

13. HINOJOSA-CUÉLLAR, J.; OLIVA-HERNÁNDEZ, J.; TORRES-HERNÁNDEZ, G.; SEGURA-CORREA, J.; ARANDA-IBÁÑEZ, E.; GONZÁLEZ-CAMACHO, J. 2012. Factores que afectan el crecimiento predestete de corderos Pelibuey en el trópico húmedo de México. Universidad y Ciencia. 28(2):163-171.

14. INSTITUTO COLOMBIANO AGROPECUARIO, ICA. 2019. Censo Pecuario Año 2019. Censo Ovino en Colombia. Disponible desde Internet en: https://www.ica.gov.co/ areas/pecuaria/servicios/epidemiologia-veterinaria/ censos-2016/censo-2018 (con acceso el 28/10/2019)

15. JAWASREH, K.; ISMAIL, Z.B.; IYA, F.; CASTAÑEDA, V.J.; VALENCIA, M. 2018. Genetic parameter estimation for pre-weaning growth traits in Jordan Awassi sheep. Veterinary World. 11(2):254-258. https://doi. org/10.14202/vetworld.2018.254-258

16. KIYANZAD, M.R. 2004. Using linear body measurements of live sheep to predict carcass characteristics for two iranian fat-tailed sheep breeds. Asian Australasian J. Anim. Sci. 17(5):93-699. https://doi.org/10.5713/ajas.2004.693

17. LEEDS, T.; MOUSEL, M.; NOTTER, D.; ZERBY, H.; MOFFET, C.; LEWIS, G. 2008. B-mode, real-time ultrasound for estimating carcass measures in live sheep: Accuracy of ultrasound measures and their relationships with carcass yield and value. J. Anim Sci. 86(11):3203-3214. https://doi.org/10.2527/jas.2007-0836

18. LOBO, R.N.B.; VILLELA, L.C.V.; LOBO, A.M.B.; PASSOS, J.R.S.; OLIVEIRA, A.A. 2006. Parámetros genéticos de características estimadas da curva de crecimiento de ovinos Santa Inés. Rev. Bras. Zootec. 35(3):1012-1018.

19. LÓPEZ-ORDAZA, R.; OLIVERA-VEGA, I.; BERRUECOSVILLALOBOSA, J.M.; PERALTA-LAILSON, M.; ULLOA-ARVIZUA, R.; VÁSQUES-PELÁEZ, C. 2012. Parámetros genéticos de pesos al nacer y al destete en ovinos de raza criolla de Chiapas. Rev. Mex. Cienc. Pecu. 3(1):113-123.

20. LUPTON, C.J. 2008. ASAS Centennial Paper: Impacts of animal science research on United States sheep production and predictions for the future. J. Anim. Sci. 86:3252-3274. https://doi.org/10.2527/jas.2008-1148

21. MACEDO, R.; ARREDONDO, V. 2008. Efecto del sexo, tipo de nacimiento y lactancia sobre el crecimiento de ovinos Pelibuey en manejo intensivo. Arch. Zootec. 57(218):219228.

22. MAZA, L.; BUSTAMANTE, M.; SIMANCA, J.; RUIZ, M.; MONTAÑO, G.; VERGARA, O. 2015. Efecto de la suplementación sobre la ganancia de peso y rendimiento en canal de corderos sudan. Rev. U.D.C.A Act. \& Div. Cient. 18(1):283-286. https://doi.org/10.31910/rudca.v18. n1.2015.448

23. MORTIMER, S.I.; HATCHER, S.; FOGARTY, N.M.; VAN DER WERF, J.H.J.; BROWN, D.J.; SWAN, A.A.; GREEFF, J.C.; REFSHAUGE, G.; HOCKING EDWARDS, J.E.; GAUNT, G.M. 2017. Genetic parameters for wool traits, live weight, and ultrasound carcass traits in Merino sheep. J. Anim. Sci. 95(5):1879-1891. https://doi.org/10.2527/ jas.2016.1234

24. NOTTER, D.R.; MOUSEL, M.R.; ZERBY, H.N.; SURBER, L.M.M.; LEEDS, T.D.; MOELLER, S.J.; LEWIS, G.S.; TAYLOR, J.B. 2014. Impact of changes in weight, fat depth, and loin muscle depth on carcass yield and value 
and implications for selection and pricing of rams from terminal-sire sheep breeds. Sheep Goat Res. J. 29:36-44.

25. PATIÑO, R.; VAN CLEEF, E. 2010. Aspectos chave do crescimento em ovinos. Rev. Col. Cienc. Anim. 2(2):399421. https://doi.org/10.24188/recia.v2.n2.2010.322

26. QUINTERO, A.; BOSCÁN, J.; PALOMARES, A.; GONZALEZ, J.; BOISSIERE, R. 1997. Efecto del sexo sobre el peso corporal a diferentes edades en corderos West-African criados en el tropico venezolano. Arch. Latinoam. Prod. Anim. 5(Supl. 1):426-427.

27. RAOOF, S.O.; MAHMUD, K.I.; ABDUL KAREEM, A.A.; MOUHAMMAD, K.G. 2017. Estimation of the best linear unbiased preduction (BLUP) of rams depending on their progeny's birth and weaning weight. The Iraqi J. of Agricul. Sci. 48(6):1405-1411.
28. RÍOS-UTRERA, A.; CALDERÓN-ROBLES, R.; LAGUNESLAGUNES, J.; OLIVA-HERNÁNDEZ, J. 2014. Ganancia de peso predestete en corderos Pelibuey y sus cruces con Blackbelly, Dorper y Katahdin. Rev. Electr. Nova Sci. 6(12):272-286.

29. SANTANA, V. 1999. Diccionario Cultural de Córdoba. Domus Libri. Bogotá (Colombia). p.285-291.

30. STATISTICAL ANALYSIS SYSTEM INSTITUTE, SAS. 2007. SAS/STAT User's guide (Relase 9.1.3), Cary, NC, USA.

31. SAFI, A.; KALERI, H.; MUHAMMAD, G.; KALERI, R.; KALERI, A.; SAFI, M.; ULLAH, A.; MANDOKHIAL, K.; SIDDIQ, M. 2017. Effect of genetic parameters on some growth performance traits of Harnai Sheep. J. of Basic \& App. Sci. 13:60-62. https://doi.org/10.6000/19275129.2017.13.11 